\title{
Role of band structure and local-field effects in the low-energy collective electronic excitation spectra of $2 \mathrm{H}$ - $\mathrm{NbSe}_{2}$
}

\author{
M. N. Faraggi, ${ }^{1}$ A. Arnau,,${ }^{1,2,3}$ and V. M. Silkin ${ }^{1,2,4}$ \\ ${ }^{1}$ Donostia International Physics Center (DIPC), Paseo de Manuel Lardizabal 4, E-20018 San Sebastián, Spain \\ ${ }^{2}$ Departamento de Física de Materiales, Facultad de Ciencias Químicas, Universidad del País Vasco, Apartado 1072, \\ E-20080 San Sebastián, Spain \\ ${ }^{3}$ Centro de Física de Materiales CFM, Materials Physics Center MPC, Centro Mixto CSIC-UPVIEHU, Paseo de Manuel Lardizabal 5 , \\ E-20018 San Sebastián, Spain \\ ${ }^{4}$ IKERBASQUE, Basque Foundation for Science, E-48011 Bilbao, Spain
}

(Received 14 March 2012; revised manuscript received 16 May 2012; published 11 July 2012)

\begin{abstract}
We present a study of the electron dynamics in the layered compound $2 \mathrm{H}-\mathrm{NbSe}_{2}$. First-principles calculations are used to obtain the band structure employed in the evaluation of the loss function with inclusion of local-field (LF) effects. Two different symmetry directions [(100) and (010)] were explored in the hexagonal basal plane. In both cases, a low-energy charge-carrier plasmon $(\mathrm{CCP})$ at $\sim 1 \mathrm{eV}$ presenting a negative dispersion over a wide momentum transfer range is found, in agreement with recent experimental results [Wezel et al., Phys. Rev. Lett. 107, 176404 (2011)]. On the contrary, in the (001) perpendicular direction, the CCP has negative dispersion at small momenta only, presenting strong positive dispersion at larger momenta. Our calculations reveal that this behavior can be explained without invoking many-body effects, as long as band structure effects are properly included in the evaluation of the excitation spectra. In addition to this CCP mode, we find another one with an arclike oscillating dispersion along the perpendicular direction, as well as the appearance of a CCP replica at high momenta due to LF effects.
\end{abstract}

DOI: 10.1103/PhysRevB.86.035115

PACS number(s): 71.45.Gm, 71.45.Lr, 78.40.Kc

\section{INTRODUCTION}

In the second part of the last century, transition-metal dichalcogenides (TMDs) were studied for different reasons. In numerous studies, these compounds attract attention because of the existence of the charge density wave (CDW) phenomenon $^{1-8}$ (like in $\mathrm{NbSe}_{2}, \mathrm{TaS}_{2}$, and $\mathrm{TaSe}_{2}$ ), while in others (like in $\mathrm{NbTe}_{2}, \mathrm{TaS}_{2}$, and $\mathrm{TaSe}_{2}$ ) the interest lies in superconducting properties. ${ }^{9-12}$ Very recently, an interesting observation on the electron dynamics in some of these compounds has been reported. ${ }^{13,14}$ By means of electron energy-loss spectroscopy (EELS) the anomalous dispersion of a collective electron excitation-plasmon-at low energies has been demonstrated. The energy of this low-energy plasmon called the charge-carrier plasmon (CCP) is at around $1 \mathrm{eV}$. In some CDW materials it shows an atypical behavior, namely, an initial negative dispersion. Moreover, the dynamical structure factor has been measured in a set of CDW materials, ${ }^{14}$ and it was found that some compounds, like $2 \mathrm{H}$-TaSe $\mathrm{T}_{2}, 2 \mathrm{H}$ $\mathrm{TaS}_{2}$, and $2 \mathrm{H}-\mathrm{NbSe}_{2}$, which experience at low temperature a charge density transition, have a negative plasmon dispersion, whereas those not showing the CDW transition, like $2 \mathrm{H}-\mathrm{NbS}_{2}$, have a positive plasmon dispersion. ${ }^{15}$ On the basis of this observation it was pointed out ${ }^{14}$ that the sign of the CCP dispersion in these materials is crucial and can be used to shed light on the importance of many-body effects.

However, it is important to follow each and every step in the determination of the full self-consistent response of the many-body system in a systematic way. This requires starting by an accurate determination of the band structure and the calculation of the noninteracting response function, as well as the inclusion of local-field ${ }^{16}$ (LF) effects when calculating the full self-consistent response (see next section).
Note that the experimental observation ${ }^{17,18}$ of negative plasmon dispersion in heavy alkali metals was naturally explained by the presence of the intraband and interband transitions. ${ }^{19,20}$ We are not aware of similar ab initio calculations explicitly devoted to the determination of the CCP plasmon dispersion in the TMDs. In the case of $2 \mathrm{H}-\mathrm{NbSe}_{2}$ such kind of investigation was only done for static susceptibility ${ }^{21}$ and in the optical limit. $^{22}$

At the same time, there is a rich literature on plasmons in other layered systems like semiconductor superlattice structures, ${ }^{23-28}$ high- $T_{c}$ oxides, ${ }^{29,30} \mathrm{MgB}_{2},{ }^{31}$ other layered superconductors, ${ }^{32,33}$ and intercalated graphite. ${ }^{34}$ In these studies it was demonstrated that the presence of the layered structure leads to strong modifications in the plasmon spectrum in comparison with that of a homogeneous three-dimensional electron gas. Thus in addition to the conventional bulk plasmon the existence in such systems of an additional mode with characteristic soundlike dispersion was demonstrated.

In this work we perform a systematic study of the dynamical electronic response properties of layered $2 \mathrm{H}-\mathrm{NbSe}_{2}$ based on a first-principles band structure calculation and taking into account LF effects. We find a strong anisotropy of the dielectric properties in this compound along the main three crystallographic directions. In particular, a strong anisotropy is observed in the CCP dispersion, which is essentially negative in the basal hexagonal plane and mainly positive in the perpendicular direction. In addition to the $\mathrm{CCP}$, we report the existence of another hitherto unknown plasmon at lower energies characterized by a peculiar arclike dispersion in the perpendicular direction. We demonstrate the crucial role of LF effects in the formation of the excitation spectra of this material at large momentum transfers, as well as a minor role of the 
exchange-correlation (XC) effects beyond the random-phase approximation.

The paper is organized as follow: In Sec. II we describe the methods used, which include a brief description of the linear response formalism and some technical details about the way the numerical calculations have been done. Section III corresponds to the analysis of the results obtained and contains a number of subsections. The conclusions are summarized in Sec. IV. Atomic units are used throughout unless otherwise stated.

\section{METHODS}

\section{A. Linear response formalism}

Information on the collective electronic excitations (plasmons) corresponding to charge density oscillations in the valence electron system can be obtained from the evaluation of the inverse dielectric function. In principle, it contains all the information about the single-particle and collective excitations of the system. To reach this goal, one needs to calculate the imaginary part of the inverse dielectric function $\epsilon^{-1}$, also known as the energy-loss function, which is related to the density-response function $\chi$ of the interacting electron system. For a periodic crystal they are related in reciprocal space through the matrix equation

$$
\epsilon_{\mathbf{G G}^{\prime}}^{-1}(\mathbf{q}, \omega)=\delta_{\mathbf{G G}^{\prime}}+V_{\mathbf{G}}(\mathbf{q}) \chi_{\mathbf{G G}^{\prime}}(\mathbf{q}, \omega),
$$

where $\omega$ is the energy transferred to the system, $\mathbf{q}$ is a wave vector in the first Brillouin zone (1BZ), $\mathbf{G}$ and $\mathbf{G}^{\prime}$ are reciprocal lattice vectors, and $V_{\mathbf{G}}(\mathbf{q})=4 \pi /|\mathbf{q}+\mathbf{G}|^{2}$ is the Fourier transform of the bare Coulomb potential. In this notation, the total momentum transfer to the system can be expressed as $\mathbf{Q}=\mathbf{q}+\mathbf{G}$ and is obtained from the knowledge of a diagonal matrix element $\epsilon_{\mathbf{G G}}^{-1}(\mathbf{q}, \omega)$. Within the framework of time-dependent density functional theory, ${ }^{35,36}$ the density-response function is defined by

$$
\begin{aligned}
\chi_{\mathbf{G G}^{\prime}}(\mathbf{q}, \omega)= & \chi_{\mathbf{G G}^{\prime}}^{0}(\mathbf{q}, \omega)+\sum_{\mathbf{G}_{1}} \sum_{\mathbf{G}_{2}} \chi_{\mathbf{G G}_{1}}^{0}(\mathbf{q}, \omega) \\
& \times\left[V_{\mathbf{G}_{1}}(\mathbf{q}) \delta_{\mathbf{G}_{1} \mathbf{G}_{2}}+K_{\mathbf{G}_{1} \mathbf{G}_{2}}^{\mathrm{xc}}(\mathbf{q}, \omega)\right] \chi_{\mathbf{G}_{2} \mathbf{G}^{\prime}}(\mathbf{q}, \omega),
\end{aligned}
$$

where $\chi^{0}$ represents the density-response function of the noninteracting electron system and $K^{\mathrm{xc}}$ contains the $\mathrm{XC}$ effects. In this work, we have performed calculations with the use of two frequently used approaches, first within the framework of the random-phase approximation (RPA) $\left(K^{\mathrm{xc}}=\right.$ $0)$ and, second, in the adiabatic local density approximation (ALDA). ${ }^{35,37}$

The noninteracting density-response function $\chi^{0}$ is determined from ${ }^{16}$

$$
\begin{aligned}
\chi_{\mathbf{G G}^{\prime}}^{0}(\mathbf{q}, \omega)= & \frac{1}{\Omega} \sum_{\mathbf{k}}^{1 \mathrm{BZ}} \sum_{n n^{\prime}} \frac{f_{n \mathbf{k}}-f_{n^{\prime} \mathbf{k}+\mathbf{q}}}{\varepsilon_{n \mathbf{k}}-\varepsilon_{n^{\prime} \mathbf{k}+\mathbf{q}}+(\omega+\mathrm{i} \eta)} \\
& \times\left\langle\psi_{n \mathbf{k}}\left|e^{-i(\mathbf{q}+\mathbf{G}) \cdot \mathbf{r}}\right| \psi_{n^{\prime} \mathbf{k}+\mathbf{q}}\right\rangle \\
& \times\left\langle\psi_{n^{\prime} \mathbf{k}+\mathbf{q}}\left|e^{i\left(\mathbf{k}+\mathbf{G}^{\prime}\right) \cdot \mathbf{r}}\right| \psi_{n \mathbf{k}}\right\rangle,
\end{aligned}
$$

being $\Omega$ the normalization volume, $\eta$ is a positive infinitesimal, and $\mathbf{k}$ 's are wave vectors in the 1BZ. Here the occupation factors $f_{n \mathbf{k}}$ are represented by a Fermi distribution function with an electronic temperature of $0.01 \mathrm{eV}$. In order to accelerate the rather time consuming calculations, instead of a direct use of expression (3) we employ the approach described by Aryasetiawan and Gunnarsson. ${ }^{38}$ It consists in the evaluation of, at the first step, the spectral function matrix using the expression

$$
\begin{aligned}
S_{\mathbf{G G}^{\prime}}^{0}(\mathbf{q}, \omega)= & \frac{1}{\Omega} \sum_{\mathbf{k}}^{1 \mathrm{BZ}} \sum_{n n^{\prime}}\left(f_{n \mathbf{k}}-f_{n^{\prime} \mathbf{k}+\mathbf{q}}\right) \delta\left(\varepsilon_{n \mathbf{k}}-\varepsilon_{n^{\prime} \mathbf{k}+\mathbf{q}}+\omega\right) \\
& \times\left\langle\psi_{n \mathbf{k}}\left|e^{-i(\mathbf{q}+\mathbf{G}) \cdot \mathbf{r}}\right| \psi_{n^{\prime} \mathbf{k}+\mathbf{q}}\right\rangle\left\langle\psi_{n^{\prime} \mathbf{k}+\mathbf{q}}\left|e^{i\left(\mathbf{k}+\mathbf{G}^{\prime}\right) \cdot \mathbf{r}}\right| \psi_{n \mathbf{k}}\right\rangle .
\end{aligned}
$$

Once the $S_{\mathbf{G G}^{\prime}}^{0}(\mathbf{q}, \omega)$ matrix is obtained on the discrete energies $\omega_{j}$ set, the imaginary part of $\chi_{\mathbf{G G}^{\prime}}^{0}\left(\mathbf{q}, \omega_{j}\right)$ is obtained from

$$
S_{\mathbf{G G}^{\prime}}^{0}\left(\mathbf{q}, \omega_{j}\right)=-\frac{1}{\pi} \operatorname{Im} \chi_{\mathbf{G G}^{\prime}}^{0}\left(\mathbf{q}, \omega_{j}\right)
$$

The real part of $\chi_{\mathbf{G G}^{\prime}}^{0}\left(\mathbf{q}, \omega_{j}\right)$ on the same set of $\omega_{j}$ 's is obtained numerically from the Hilbert transformation.

\section{B. Numerical calculations}

The precise evaluation of $\chi^{0}$ is a key factor to obtain reliable results in the low-energy domain of all quantities depending on it. For this purpose, the noninteracting density-response function is calculated through Eq. (4) involving the summation over the $1 \mathrm{BZ}$ in a fine $96 \times 96 \times 16 \mathbf{k}$ mesh. The second sum in Eq. (4), running over $n$ and $n^{\prime}$, refers to the band index and contains all occupied and unoccupied valence states up to an energy of $50 \mathrm{eV}$ above the Fermi level. In the numerical computation the delta function in Eq. (4) is replaced by a Gaussian with a $0.025 \mathrm{eV}$ broadening.

In order to calculate all magnitudes of interest described above, we use the single-electron states obtained by means of density functional theory with an exchange-correlation potential in the Ceperley-Alder form ${ }^{39}$ with the PerdewZunger parametrization. ${ }^{40}$ The corresponding one-electron energies $\varepsilon_{n \mathbf{k}}$ and wave functions $\psi_{n \mathbf{k}}$ are obtained using an energy cutoff in the plane wave basis set of $540 \mathrm{eV}$ in the self-consistent electronic structure calculations. The niobium and selenium ion-electron interactions are described by normconserving pseudopotentials constructed according to Ref. 41. $2 \mathrm{H}-\mathrm{NbSe}_{2}$ crystallizes in a layered hexagonal structure with the niobium atoms at the center of trigonal selenium prisms. The corresponding projection on the basal hexagonal plane is shown in Fig. 1(a). Experimental lattice parameters ${ }^{42}$ were employed in the calculations [in the direction perpendicular to the basal hexagonal layers the parameter is $c=12.55 \AA$ (interlayer spacing between $\mathrm{Nb}$ planes equals $c / 2$ ), while that laying in the plane is $a=3.44 \AA]$. The Se ions planes were located along the $c$ axis at $z=0.1183 c$, as in Ref. 21 .

Local-field effects in the theory are taken into account through the inclusion of nondiagonal elements in the calculation of the $\chi_{\mathbf{G G}^{\prime}}(\mathbf{q}, \omega)$ matrices. The plane wave expansion was used taking into account up to 40 plane waves. The results when only intraband transitions are considered were obtained by including only the terms with $n=n^{\prime}$ in the summation over the energy bands in Eq. (3). In this case only three energy 

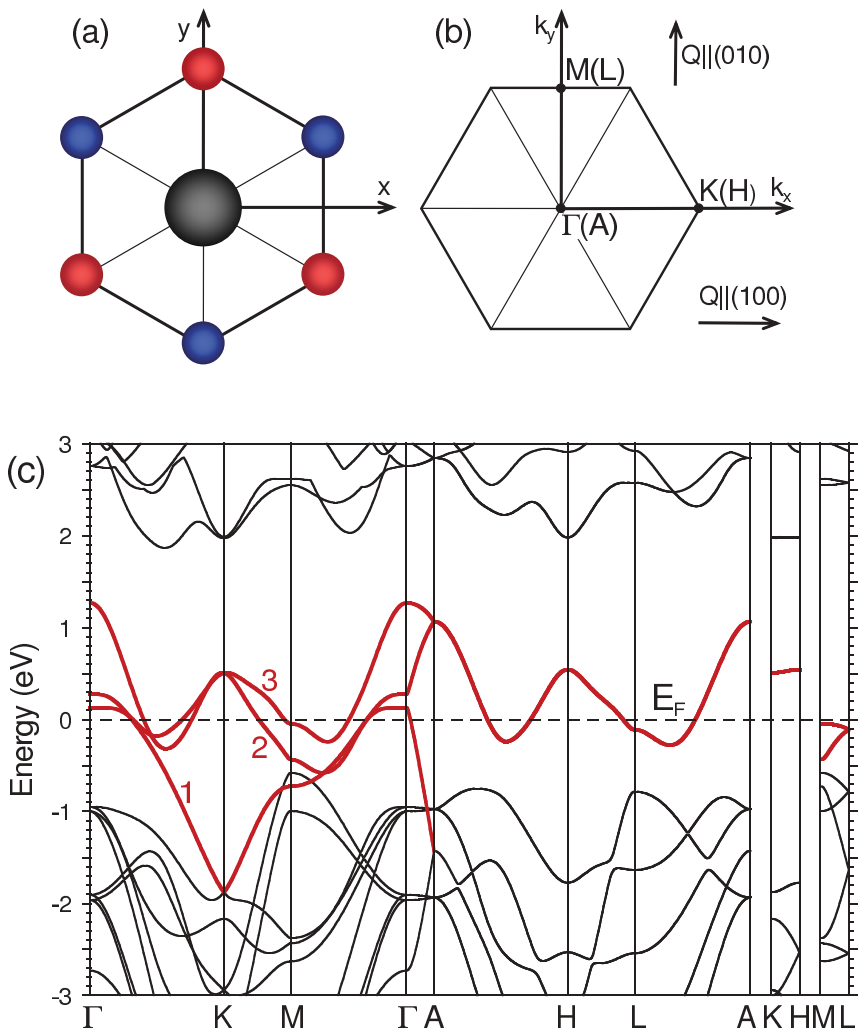

FIG. 1. (Color online) (a) Projection onto a hexagonal basal plane of atomic positions of the $2 \mathrm{H}-\mathrm{NbSe}_{2}$ unit cell. The $\mathrm{Nb}$ ion positions at $z=c / 4$ and $z=-c / 4$ are shown by the large gray filled circle. The Se ions are shown by smaller filled circles. The planes containing the Se ions located at $z=0.1183 c$ and $z=-0.3817 c(z=-0.1183 c$ and $z=0.3817 c$ ) are shown by red (blue) color circles. (b) The first Brillouin zone (1BZ) with main symmetry directions for the in-plane momentum transfer. The (001) axis is directed in the perpendicular direction. The ГKM (AHL) plane is located at $k_{z}=0\left(k_{z}=\pi / c\right)$. In this paper we use the convention wherein the (100), (010), and (001) momentum transfer axes are directed along the $\Gamma \mathrm{K}, \Gamma \mathrm{M}$, and $\Gamma \mathrm{A}$ directions of the 1BZ, respectively. (c) The calculated band structure along some symmetry directions of the $1 \mathrm{BZ}$ around the Fermi level which set at the zero energy. The three energy bands in which the intraband transitions are available are shown by thick red lines and labeled as 1,2 , and 3 .

bands crossing the Fermi level are involved in the evaluation of $\chi^{0}$.

\section{RESULTS AND DISCUSSION}

\section{A. Electronic band structure}

We begin this section with the presentation of the electronic structure of $2 \mathrm{H}-\mathrm{NbSe}_{2}$ in Fig. 1(c). The calculated electronic band structure is in fairly good agreement with the $a b$ initio band structures obtained by other methods ${ }^{21,22,43-46}$ and measured in angle-resolved photoemission experiments. ${ }^{46-50}$ Some observed differences from the previous calculations performed using other methods are at the same level as the differences among them. We do not expect that this uncertainty in the determination of the band structure could lead to notable effects on the calculated excitation spectra. From the band structure it is clear that the excitation spectrum in the low-energy domain is mainly determined by transitions within the three energy bands labeled as 1, 2, and 3 around the Fermi level which, as shown below, are responsible for different intraand interband transitions in the energy-loss spectra. These bands crossing each other around the Fermi surface originate from the $\mathrm{Se} 4 p$ [labeled by 1 in Fig. 1(c)] and $\mathrm{Nb} 4 d$ orbitals [labeled by 2 and 3 in Fig. 1(c)]. Their character was previously analyzed and discussed in detail by other authors. ${ }^{21,44,45}$

\section{B. Full calculation: Intraband and interband transitions}

In the following, we present results for the energy-loss function, $-\operatorname{Im}\left[\epsilon^{-1}(Q, \omega)\right]$, obtained at the RPA level, for three directions of momentum transfer. Namely, Figs. 2, 3, and 4 show $-\operatorname{Im}\left[\epsilon^{-1}(Q, \omega)\right]$ for momentum transfers along the (100), (010), and (001) symmetry directions, respectively. These symmetry directions coincide with the symmetry directions $\Gamma \mathrm{K}, \Gamma \mathrm{M}$, and $\Gamma \mathrm{A}$ in the $1 \mathrm{BZ}$. In these figures panels (a) show the energy-loss function calculated with the full inclusion of the LF effects, while in panels (b), the data were obtained without its inclusion. In all these three figures one can observe a well-defined peak in the energy-loss function at an energy $0.95 \mathrm{eV}$ for small momenta, corresponding to the CCP. The energy of this peak at small momenta is in good agreement with the value of $\sim 1 \mathrm{eV}$ obtained in optical ab initio calculation ${ }^{22}$ and measured in the energy-loss experiment. ${ }^{14,51}$

As the value of the momentum transfer increases, the dispersion of this CCP peak is remarkably different for momenta laying in the basal plane and in the perpendicular direction. This result demonstrates that the negative dispersion of the CCP peak for momenta in the basal plane can be rationally explained as being produced by the electronic structure of $\mathrm{NbSe}_{2}$. A closer inspection of the CCP peak dispersion along the (100) and (010) directions reveals that, indeed, a second peak structure marked by an arrow in Figs. 2(a) and 3(a) is observed at slightly higher energies than the CCP peak in both symmetry directions. This second branch disperses upwards almost linearly and can be detected up to energies of $\sim 2 \mathrm{eV}$ at $Q \sim 0.8 \AA^{-1}$. Comparison of Figs. 2(a) and 3(a) shows a slight difference in the spectral strength of this satellite feature. The peak is somewhat sharper for momentum transfers in the (010) direction. The origin of this feature can be assigned to the enhanced number of the interband transitions with slightly lower energies, reflected in the appearance of a peak in $\operatorname{Im}[\epsilon(q, \omega)] \equiv \operatorname{Im}\left[\epsilon_{\mathbf{G}=0, \mathbf{G}^{\prime}=0}(q, \omega)\right]$ marked by an arrow in Figs. 2(c) and 3(c).

As an example, in Fig. 5 we present the corresponding quantities calculated at the momentum transfer oriented along the (100) symmetry direction with $Q=0.152 \AA^{-1}$. Here, one can see how the presence of an additional interband peak in the imaginary part of the dielectric function at energy of $1.1 \mathrm{eV}$ produces a notable drop in the real part in the nearby energy region. As a result, in the energy-loss function $\operatorname{Im}\left[\epsilon^{-1}\right]$, apart from the dominating $\mathrm{CCP}$ peak located at $\omega=0.88 \mathrm{eV}$, a well-defined peak at $\omega=1.18 \mathrm{eV}$ appears. A similar effect can be seen at higher energies as well, where the $\omega \sim 2.6 \mathrm{eV}$ peak in $\operatorname{Im}[\epsilon]$ leads to the appearance of a broad peak structure around $3 \mathrm{eV}$ in the energy-loss function.

Comparison of the CCP dispersion of Figs. 2(a) and 3(a) with that presented in Fig. 4(a) reveals its strikingly different 

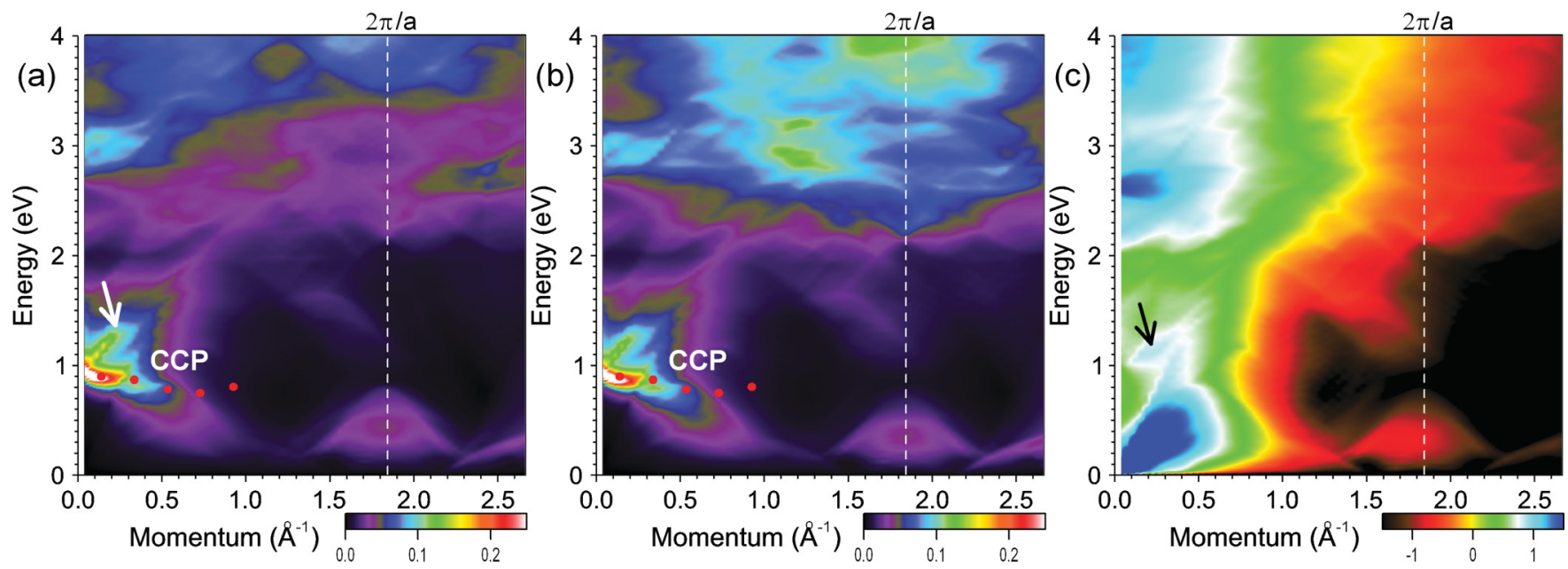

FIG. 2. (Color online) 2D plots of calculated energy-loss function of $2 H-\mathrm{NbSe}_{2}$ versus momentum transfer $Q$ along the (100) symmetry direction and energy $\omega$, calculated with (a) inclusion and (b) without inclusion of local-field effects. The data are obtained with the RPA kernel. The dominating peak corresponding to the charge-carrier plasmon (CCP) is highlighted by the corresponding symbol. The experimentally measured (Ref. 14) energy positions of peaks corresponding to the CCP are shown by filled circles. The imaginary part of $\epsilon(Q, \omega)$ evaluated with inclusion of both intraband and interband transitions is presented in (c) on a logarithmic scale. Arrow in (a) shows a satellite plasmon peak having its origin in the enhanced interband transitions peak in $\operatorname{Im}[\epsilon]$ marked by an arrow in (c).

dispersion in the (001) direction. First of all, in Fig. 4(b) it is seen that in the calculations without inclusion of the LF effects the CCP frequency at small momenta is lower by $\sim 0.1 \mathrm{eV}$ in this case. Only inclusion of such effects bring its energy to the same value as in the basal plane. Second, along the (001) direction the region where the CCP dispersion is negative is substantially smaller and for all the momentum transfers exceeding $\sim 0.1 \AA^{-1}$ its dispersion is positive and disperses up to an energy of $\sim 1.6 \mathrm{eV}$ at $Q=0.7 \AA^{-1}$.

Concerning the comparison with available data, we put in Figs. 2 and 3 the experimentally determined CCP peak positions for momentum transfers in both the (100) and (010) directions, although there exists a controversy with the definition of the basal plane momentum directions. ${ }^{13,14}$ From Figs. 2(a) and 3(a) one can see that the calculated CCP dispersion for momenta up to $Q \sim 0.6 \AA^{-1}$ is almost isotropic and has initially a negative dispersion, which is in excellent agreement with the experimental data. ${ }^{14}$ However, for momenta larger than $Q \sim 0.6 \AA^{-1}$ a good agreement with experiment is observed for momenta in the (010) direction only, whereas in the (100) direction the calculated peaks are located at somewhat lower energies. In the experimental curves of Ref. 14 shoulders are observed in the upper-energy side of the main CCP peak. However, a direct comparison is not possible and calls for a more careful analysis of the experimental data to clarify this issue.

\section{Calculations with intraband transitions only}

In order to understand this peculiar strongly anisotropic CCP dispersion, we have also calculated the energy-loss function including only intraband transitions in the summation appearing in the definition of the noninteracting response function $\chi^{0}$ of Eq. (3). The corresponding energy-loss spectra
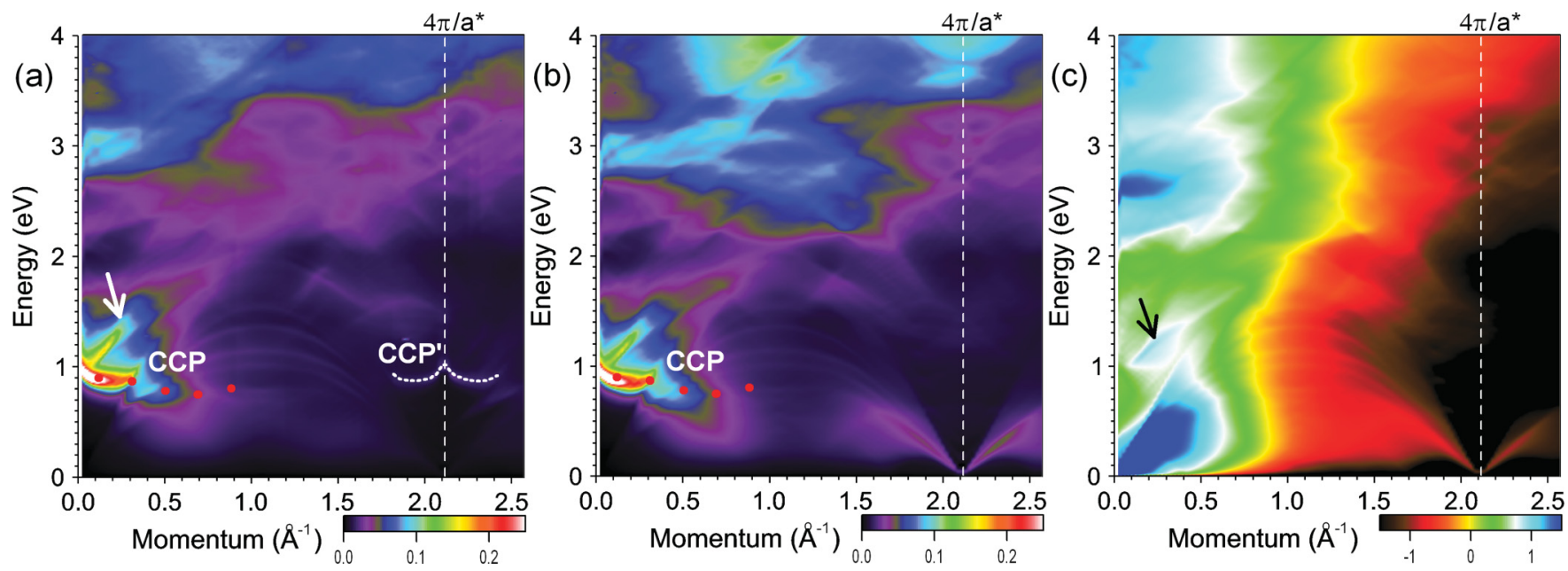

FIG. 3. (Color online) Similar to Fig. 2 for the (010) symmetry direction. Here, $a^{*}=a \sqrt{3}$. 

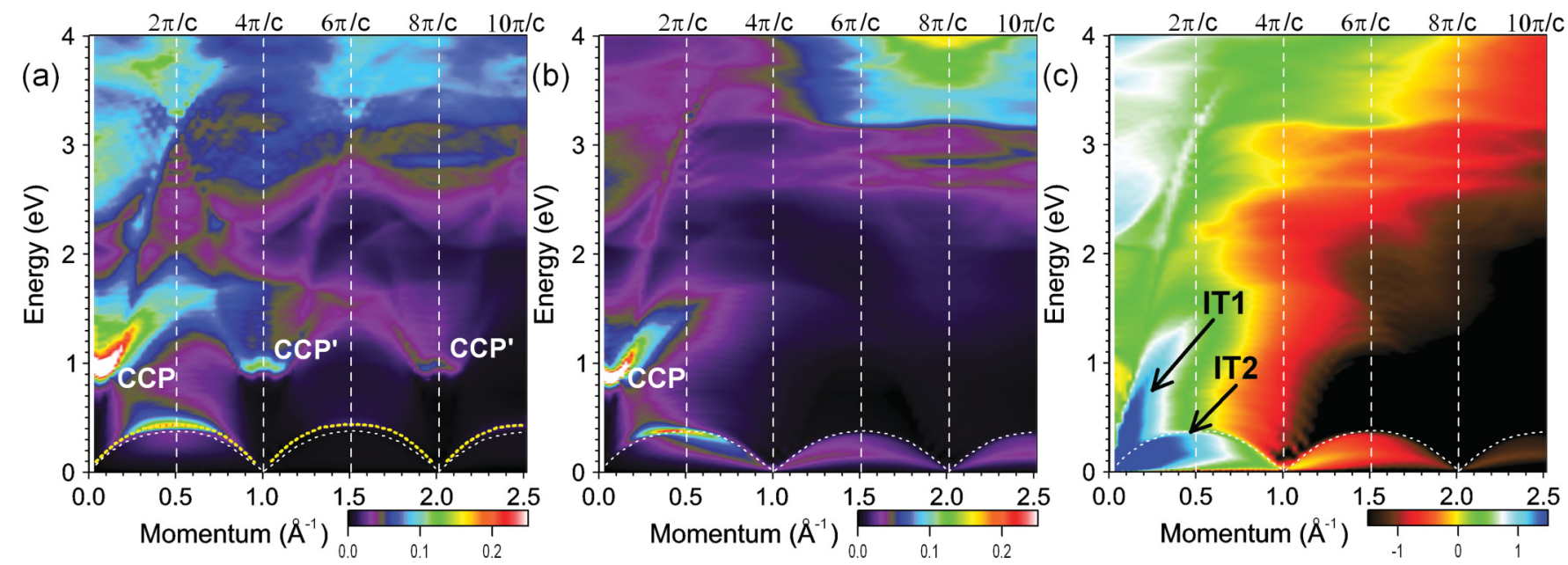

FIG. 4. (Color online) Similar to Fig. 2 for the (001) symmetry direction. In (a) the acoustic plasmon arclike dispersion is highlighted by thick dotted lines. Thin short-dashed lines delimit regions for intraband transitions within the Nb-originated bands labeled as 2 and 3 in Fig. 1(c). In (c) peaks in $\operatorname{Im}[\epsilon]$ due to intraband transitions within the Se-originated band 1 and the Nb-derived bands 2 and 3 are marked as IT1 and IT2, respectively.

and $\operatorname{Im}[\epsilon]$ calculated along the main symmetry directions of momentum transfer are presented in Figs. 6, 7, and 8. Here, we see that the inclusion of intraband transitions only in the evaluation of $\chi^{0}$ dramatically modifies the excitation spectra. At small momenta the main effect, comparing with the full calculations, consists in a large upward shift of the CCP peak in the energy-loss function from $\sim 0.95 \mathrm{eV}$ to $\sim 3.5 \mathrm{eV}$. Apart from this, a strong negative $\mathrm{CCP}$ peak dispersion is observed for all momentum transfers in all three symmetry directions. Furthermore, the CCP peak presents no damping broadening and reduces its energy from $3.5 \mathrm{eV}$ at $Q=0$ down to, e.g., $1.4 \mathrm{eV}$ at $Q=0.5 \AA^{-1}$ along the (100) direction, where it enters the electron-hole pair continuum region and acquires a finite width. Even being inside this region, the CCP

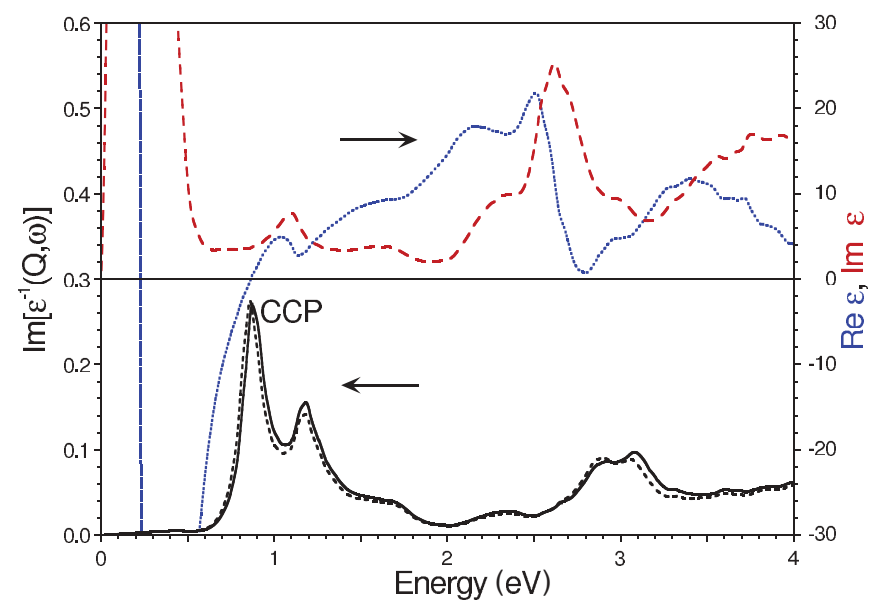

FIG. 5. (Color online) Imaginary (long-dashed line) and real (dotted line) parts of the dielectric function $\epsilon$ and corresponding energy-loss function $\operatorname{Im}\left[\epsilon^{-1}\right]$, with inclusion (solid line) and without (dashed line) inclusion of the local-field effects, calculated for momentum transfer $Q=0.152 \AA^{-1}$ along the (100) symmetry direction. Data obtained with RPA kernel are presented. The ALDA curves are undistinguished from the RPA ones on the presented scale. peak continues with a negative dispersion and its energy falls down to $\omega \sim 0.2 \mathrm{eV}$ around $Q=1 \AA^{-1}$. A similar behavior is observed in the (010) direction when LF effects are not included, as seen in Fig. 7(b). However, the picture changes appreciably when LF effects are taken into account. Figure 7(a) reveals that the $\mathrm{CCP}$ dispersion below $\sim 1.5 \mathrm{eV}$ is significantly distorted by the presence of the second plasmon peak located at lower energies. Concerning the (001) direction one can also see how the CCP peak reduces its energy down to $1.1 \mathrm{eV}$ at $Q=0.25 \AA^{-1}$ where it quickly disappears entering the electron-hole pair continuum region.

This strong negative CCP dispersion in the "intraband" case is explained by the unusually fast drop in the strength of the intraband peak in the imaginary part of dielectric function with momentum increase. We assign this behavior to the strong variation with momentum of some matrix elements required to evaluate $\chi^{0}$ of Eq. (3). These are those matrix elements involving intraband transitions within the three energy bands crossing the Fermi surface. Comparison of Figs. 2, 3, and 4 with Figs. 6, 7 , and 8 shows that the inclusion of interband transitions partially compensates for this effect. In particular, in the (001) direction it changes the $\mathrm{CCP}$ dispersion from negative to positive on a wide range of momentum transfers. However, in the (100) and (010) directions the interband transitions cannot compensate the strong reduction of spectral weight in a large range of momentum transfers and the $\mathrm{CCP}$ dispersion remains negative. Only for momenta along the (010) direction the combined effect of inclusion of interband transitions and the LF effects is able to turn the CCP dispersion to be slightly positive at $Q \geqslant$ $0.6 \AA^{-1}$ in agreement with experiment. ${ }^{14}$ In the (100) direction we do not observe any similar change in the CCP dispersion.

\section{Beyond 1BZ: Plasmon replica}

Examination of the energy-loss function for momentum transfers beyond the $1 \mathrm{BZ}$ permits us to observe a singular behavior of the CCP peak. In Figs. 2(a) and 3(a) it is seen how it disappears at $Q$ 's around $1 \AA^{-1}$. However, in Fig. 3(a) 

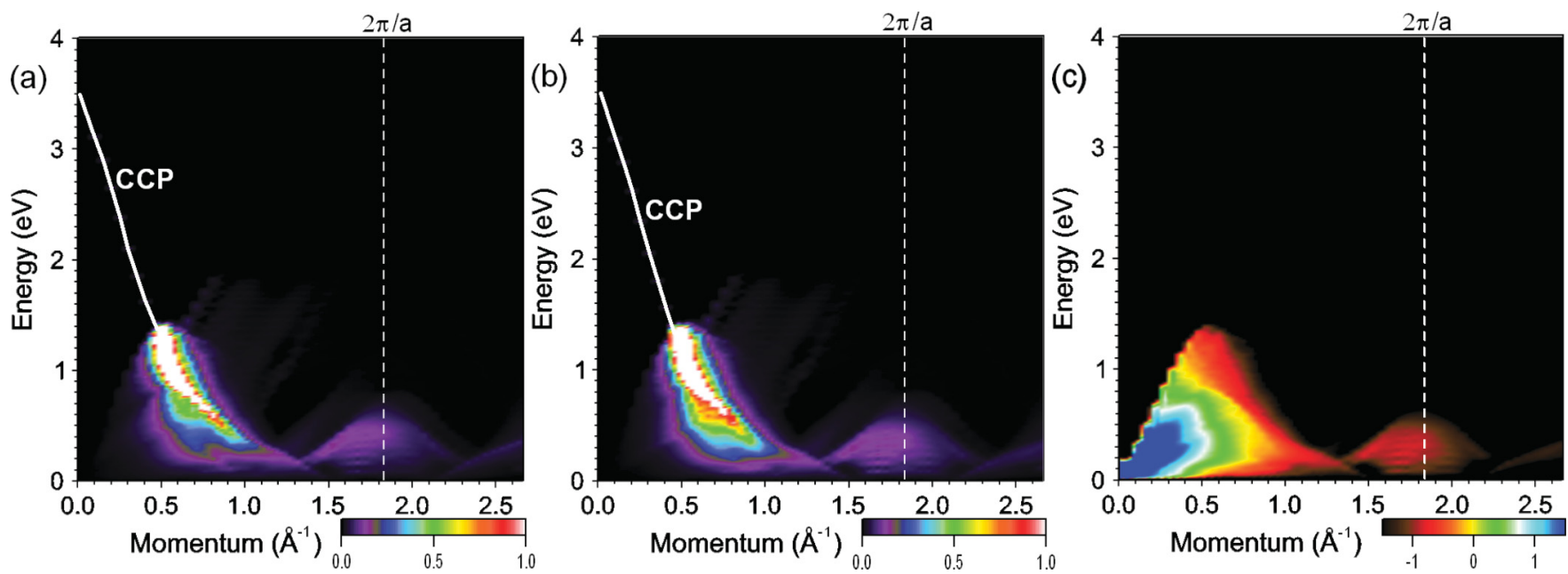

FIG. 6. (Color online) 2D plots of calculated energy-loss function of $2 H-\mathrm{NbSe}_{2}$ versus momentum transfer $Q$ along the (100) symmetry direction and energy $\omega$, calculated with (a) inclusion and (b) without inclusion of local-field effects. The intraband transitions are only included in the evaluation of $\chi^{0}$. The data are obtained with the RPA kernel. A dominating peak corresponding to the charge-carrier plasmon (CCP) is highlighted by the corresponding symbol. Imaginary part of $\epsilon(Q, \omega)$ is presented in (c) on a logarithmic scale.

one sees how this peak, labeled as $\mathrm{CCP}^{\prime}$, appears again as $Q$ approaches a value close to the value of the reciprocal lattice vector $\mathbf{G}=4 \pi / a \sqrt{3}$. In this figure it is also illustrated how, starting from the $Q=4 \pi / a \sqrt{3}$ point, the $\mathrm{CCP}^{\prime}$ dispersion repeats the $\mathrm{CCP}$ dispersion for both increasing and decreasing momentum directions. Figure 3(b) also shows that such a peak does not appear in the same energy-momentum phase-space region when the LF effects are not included. This can be seen with more detail in Fig. 11, where the data for $Q=2.02 \AA^{-1}$ along the (010) symmetry direction are reported. Here we see that in the absence of LF effects, at energies close to $0.9 \mathrm{eV}$ there is not any signature of the $\mathrm{CCP}^{\prime}$ peak in the energy-loss function.

However, when the LF effects are included, a clear $\mathrm{CCP}^{\prime}$ peak is presented in the energy-loss function. From Fig. 5 it is clear that there is some feedback to small $Q$ 's, where the inclusion of the LF effects leads to an upward shift in energy of the CCP peak. A similar reappearance of the plasmon peak at momentum values close to the reciprocal lattice vectors was obtained in the excitation spectra of compressed lithium. ${ }^{52}$ In the case of $\mathrm{MgB}_{2}$ the impact of the LF effects along the (001) symmetry direction is even stronger and an analog ${ }^{53,54}$ of the $2 \mathrm{H}-\mathrm{NbSe}_{2} \mathrm{CCP}$ with the energy of $2.5 \mathrm{eV}$ has a continuous dispersion for all momentum transfers in the perpendicular direction. ${ }^{55,56}$ On the contrary, in the case of $\mathrm{MgB}_{2}$ for momenta in the hexagonal basal plane the interband transitions are very efficient destroying this CCP-analog mode. In consequence, its replicas at larger momenta do not appear with clarity similar to that we observe in the $\mathrm{NbSe}_{2}$ case. This effect is attributed to the fact that the material is strongly nonhomogeneous in the three main symmetry directions and was carefully analyzed in detail in other systems. ${ }^{52,55}$ Therefore, we can deduce that a wide range of materials could present prominent features in the excitation spectra induced by the LF effects at large momentum transfers with magnitude exceeding the $1 \mathrm{BZ}$.
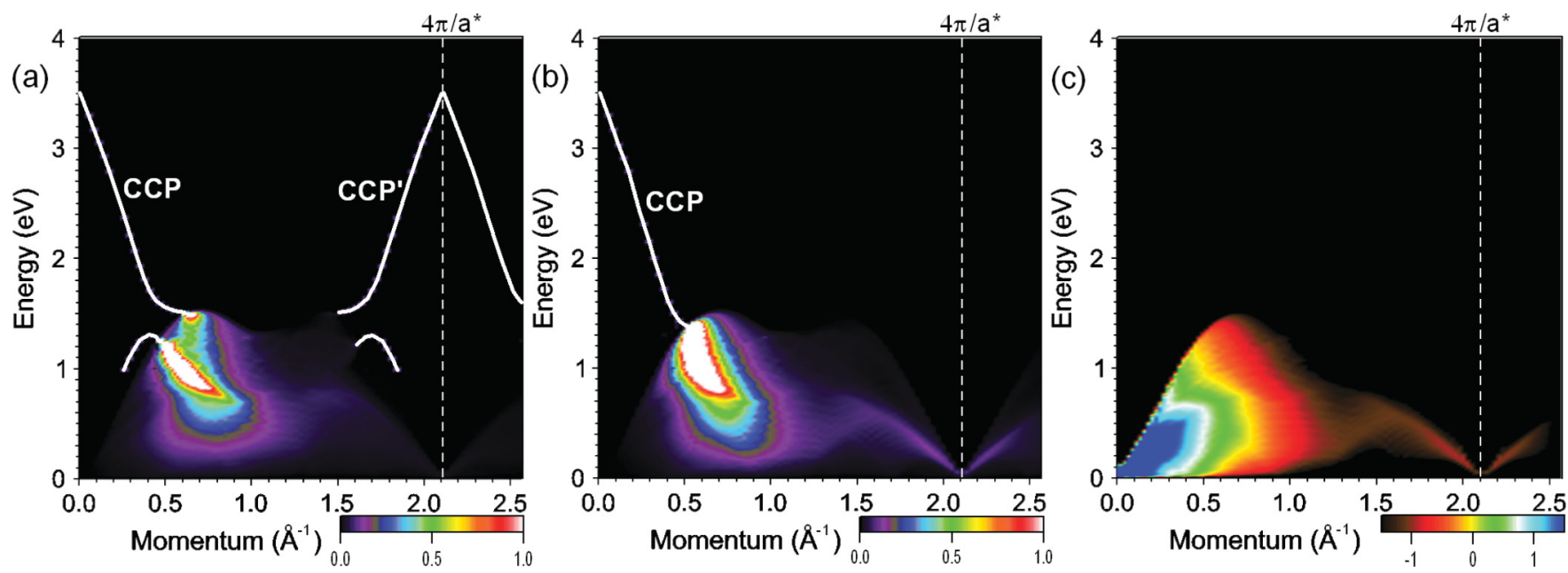

FIG. 7. (Color online) Similar to Fig. 6 for (010) symmetry direction. Here, $a^{*}=a \sqrt{3}$. 

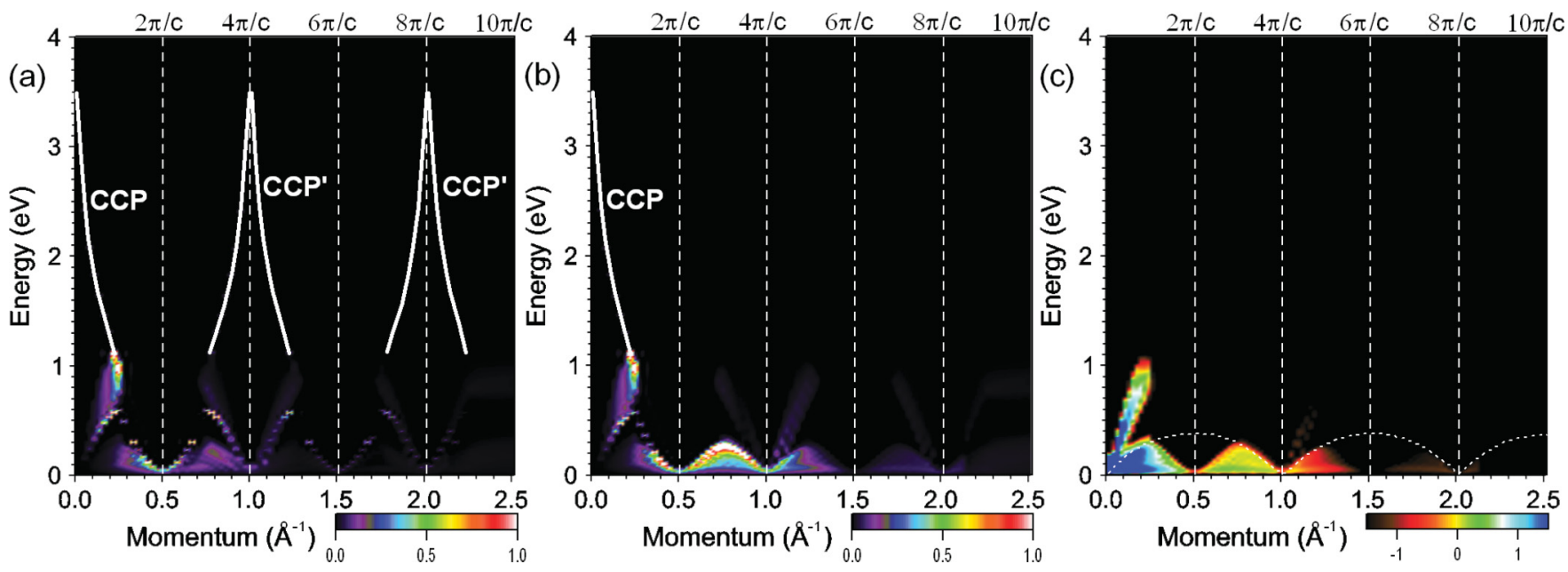

FIG. 8. (Color online) Similar to Fig. 6 for (001) symmetry direction.

\section{E. Role of LF effects}

Comparison of Figs. 3(a) and 3(b) demonstrates that the role of LF effects is clearly dependent on the energy and kind of plasmons involved. One can see how, in the absence of LF effects, the strong low-energy peak starts at $Q=4 \pi / a \sqrt{3}=$ $2.11 \AA^{-1}$ from zero energy with a quasilinear dispersion, due to a prominent peak in $\operatorname{Im}[\epsilon]$ [see Fig. 3(c)] originating from the intraband transitions. It is almost completely suppressed by the LF effects as seen in Fig. 3(a). To be more specific, in the particular case of $Q=2.02 \AA^{-1}$, this can be observed in Fig. 11: A well-defined low-energy peak with energy $0.15 \mathrm{eV}$ seen in the energy-loss function evaluated without LF effects is dramatically reduced when LF effects are included.

Coming back to Figs. 2, 3, and 4 one can observe that the differences in the excitation spectra between the (100) and (010) directions and the (001) direction are even more profound in the low-energy region. In the (001) direction we observe a low-energy peak dispersing initially with almost linear dispersion at small momenta. ${ }^{57}$ We relay the appearance of this peak to the existence of a low-energy acoustic plasmon, also predicted to exist on base of the first-principles calculations in other systems such as $\mathrm{MgB}_{2}$ and $\mathrm{Pd} .{ }^{31,58}$ Previously such kind of plasmon was intensively investigated in various layered systems ${ }^{23-34}$ and other materials with two kinds of carriers at the Fermi level. ${ }^{59-64}$ The appearance of this plasmon is related to the presence of two kinds of charge carriers characterized by different maximal Fermi velocity components in a given direction in the energy bands crossing the Fermi surface. While in the (100) and (010) directions we observe only one broad peak in the low-momentum and low-energy corners of Figs. 2(c) and 3(c), along the (001) direction the situation is qualitatively different [see Fig. 4(c)].

In this case, one can clearly resolve in $\operatorname{Im}[\epsilon]$ two intraband peaks denoted as IT1 and IT2 which disperse initially with very different group velocities in this energy-momentum phase-space region. The peak IT1 originates from the intraband transitions within the Se-derived band 1, while the peak IT2 has its origin in the intraband transitions within the $\mathrm{Nb}$-derived bands 2 and 3 with a more slow dispersion in this direction. As a consequence of this two-peak structure in $\operatorname{Im}[\epsilon]$, the corresponding real part suffers dramatic modifications, as compared to the one expected from the free-electron gas model. ${ }^{65}$ More precisely, instead of crossing once the zero axis, the real part does it three times, as illustrated by the circles in Fig. 9 for $Q=0.189 \AA^{-1}$. However, only one of them, in combination with a local minimum in $\operatorname{Im}[\epsilon]$, produces a peak in the energy-loss function, i.e., highlighting the existence of a well-defined collective excitation. Note that in Fig. 9 the fourth zero crossing of $\operatorname{Re}[\epsilon]$ at $\omega=1 \mathrm{eV}$ leads to appearance of the $\mathrm{CCP}$ peak in the energy-loss function at slighter higher energy.

From Fig. 4 it is clear that the role of the LF effects is even more remarkable along the (001) direction. On the one hand, in the same fashion as occurs at momentum transfers laying in the basal plane, LF effects produce the appearance of the $\mathrm{CCP}^{\prime}$ replicas at $\sim 0.95 \mathrm{eV}$ energy for momentum transfers with values close to the reciprocal lattice vectors $Q=4 \pi / c$, $Q=8 \pi / c$, and probably for larger vectors as well. On the

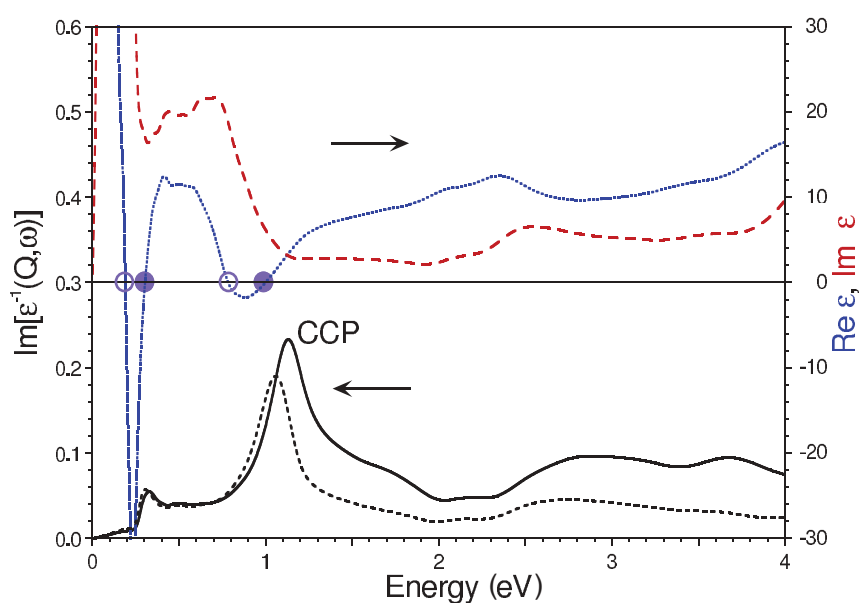

FIG. 9. (Color online) Similar to Fig. 5 calculated for momentum transfer $Q=0.189 \AA^{-1}$ along the (001) symmetry direction. Circles highlight the zero crossing in the real part of $\epsilon$. Only the zero crossings denoted by filled dots produce a peak in the energy-loss function, whereas the crossings shown by open circles do not produce any effect due to peaks in the imaginary part of $\epsilon$ at fairly the same energies. 


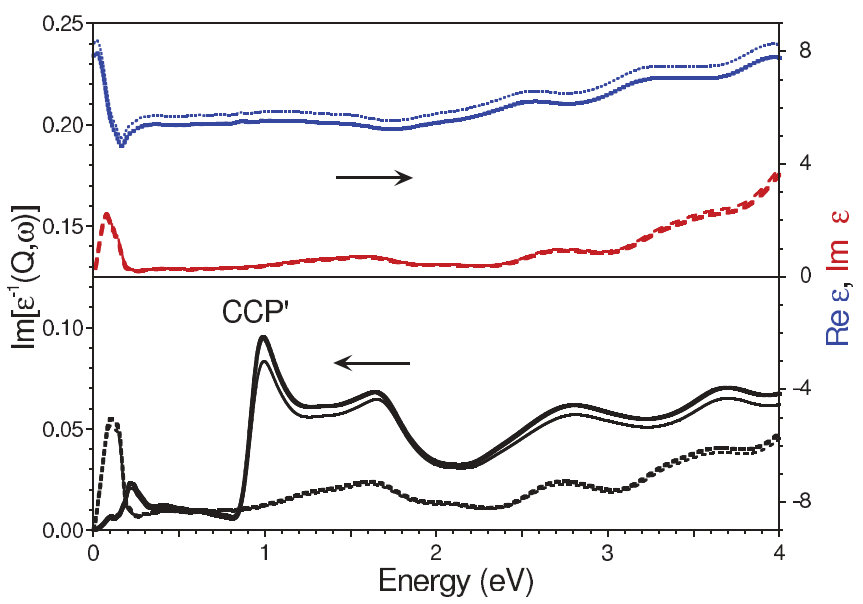

FIG. 10. (Color online) Similar to Fig. 5 calculated for momentum transfer $Q=0.881 \AA^{-1}$ along the (001) symmetry direction. Thin (thick) lines stand for the data obtained in RPA (ALDA) calculations.

other hand, in the lower energy part its role is completely different: Instead of a suppression of low-energy peaks the LF effects are responsible for the transmittance of a low-energy acoustic-like plasmon with an arclike oscillating dispersion, very similarly to what occurs in $\mathrm{MgB}_{2} \cdot{ }^{31}$

From comparison of Figs. 4(a) and 4(b) one can see how the spectral weight from the energy-loss function at small $Q$ 's is transmitted to the higher energies beyond the regions limited by thin short-dashed lines that correspond to intraband transitions within the bands 2 and 3 (region IT2 and its replicas at larger $Q$ 's). In this particular case the acoustic plasmon arclike dispersion appears at slightly higher energies beyond these regions. This ensures that plasmons cannot decay into intraband transitions in the energy bands with lower Fermi velocities in the perpendicular direction and have a rather long lifetime. Some details of this behavior can be observed in Fig. 10. Here it is seen how at $Q=0.881 \AA^{-1}$ the sharp peak at $\omega=0.12 \mathrm{eV}$ in the energy-loss function evaluated without inclusion of the LF effects repeats just the peak in the imaginary part of $\epsilon$ which in turn reflects an enhanced number of incoherent electron-hole pairs which can be created in the bands 2 and 3. However, when the LF effects are included this peak in the energy-loss function is strongly suppressed and instead another peak at $\omega=0.22 \mathrm{eV}$ appears, at the energy where exactly the acoustic plasmon exists at $q=4 \pi / c-Q$.

\section{F. XC effects}

The role of the XC effects in the formation of the excitation spectrum of solids has attracted attention for many years. There have been numerous attempts to explain the observed deviations in the measured data from predictions based on freeelectron-like models to gauge the importance of XC effects. However, in many cases of systems with moderate correlation effects, the observations could be reasonably explained when the band structure effects were included at the $a b$ initio level. As mentioned above, we obtained here two sets of results with RPA and ALDA kernels. From a careful analysis of the data we can conclude that the role played by the XC effects beyond

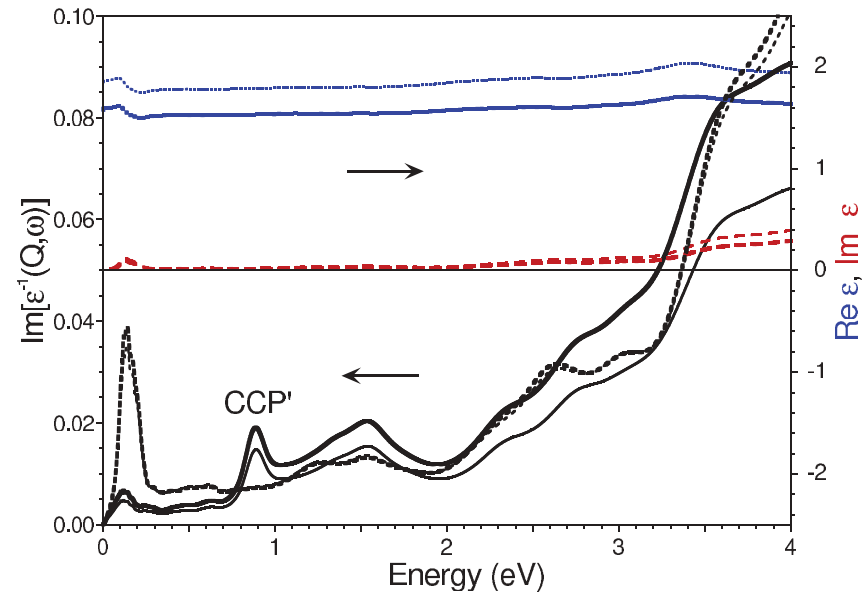

FIG. 11. (Color online) Similar to Fig. 5 calculated for momentum transfer $Q=2.02 \AA^{-1}$ along the (010) symmetry direction. Thin (thick) lines stand for the data obtained in RPA (ALDA) calculations.

RPA in this compound is rather small, at least in the energy region of interest here. For small values of the momentum transfer this role is negligible, as is confirmed in Figs. 5 and 9 , which is in line with previous observations. ${ }^{22}$ At the large momentum transfers its role gradually increases and some modifications in the excitation spectra can be detected.

Thus, in Fig. 11 one can see how at $Q=2.02 \AA^{-1}$ along the (010) direction the XC effects produce an upward shift in the energy-loss function, being more efficient at larger energies. Similar small effects of inclusion of the ALDA kernel on the excitation spectra can also be seen in Fig. 10 for $Q$ along the (001) symmetry direction. Nevertheless, in general, the effect of such inclusion is not large and produces only some redistribution in the absorption strength but does not lead to qualitative changes in the excitation spectra.

\section{CONCLUSIONS}

We have shown the existence of a picture rich in spectral features when studying the low-energy dielectric properties of the layered transition metal niobium diselenide. Many interesting effects related to the band structure have been demonstrated to exist in this material in addition to those recently observed. ${ }^{14}$ Our results demonstrate that the chargecarrier plasmon dispersion in the hexagonal basal plane is negative over the large range of momentum transfers, while it quickly changes its dispersion from the initially negative to positive in the perpendicular direction. The calculation of the dielectric function extended over a momentum range outside the first Brillouin zone permits us to demonstrate the importance of local field effects in the formation of excitation spectra at large $Q$ values and the unimportance of XC effects, particularly at low energy and low momentum transfers. In particular, we find replicas of the CCP at large momenta due to local-field effects, which is a behavior very similar to the one described for Li under pressure. ${ }^{52}$

In the low-energy domain, in addition to the charge-carrier plasmon, we find a plasmon which exists only for momentum transfers along the perpendicular direction but it does not appear in the basal plane. Furthermore, it possesses a peculiar 
arclike oscillating dispersion with energy below $0.45 \mathrm{eV}$ which resembles an acoustic-like plasmon recently found in $\mathrm{MgB}_{2}{ }^{31}$

In summary, we do not find any contradiction between the experimentally determined ${ }^{14}$ plasmon dispersion and the one obtained in our calculations, as long as the realistic band structure information is properly included. Furthermore, our results for $2 \mathrm{H}-\mathrm{NbSe}_{2}$ are a clear demonstration of the inadequacy of using predictions based on the free-electron gas model to describe valence electron excitations in this kind of system. Hence, the CCP dispersion data in this case can be naturally explained only by band structure effects and call for similar studies in other transition-metal dichalcogenides.

\section{ACKNOWLEDGMENTS}

This work was supported in part by the Basque Departamento de Educación, Universidades, e Investigación (Grant No. IT-366-07) and the Spanish Ministerio de Ciencia e Innovación (Grant No. FIS2010-19609-C02-00).
${ }^{1}$ J. A. Wilson, F. J. DiSalvo, and S. Mahajan, Adv. Phys. 24, 117 (1975).

${ }^{2}$ D. E. Moncton, J. D. Axe, and F. J. DiSalvo, Phys. Rev. Lett. 34, 734 (1975).

${ }^{3}$ J. M. E. Harper, T. H. Geballe, and F. J. Di Salvo, Phys. Lett. A 54, 27 (1975).

${ }^{4}$ T. M. Rice and G. K. Scott, Phys. Rev. Lett. 35, 120 (1975).

${ }^{5}$ N. J. Doran, B. Ricco, M. Schreiber, D. Titterington, and G. Wexler, J. Phys. C: Solid State Phys. 11, 699 (1978).

${ }^{6}$ S.-K. Chan and V. Heine, J. Phys. F: Met. Phys. 3, 795 (1973).

${ }^{7}$ G. Grüner, Density Waves in Solids, Vol. 89 of Frontiers in Physics (Addison-Wesley, Reading, MA, 1994).

${ }^{8}$ K. Rossnagel, J. Phys.: Condens. Matter 23, 213001 (2011).

${ }^{9}$ M. H. Van Maaren and G. M. Schaeffer, Phys. Lett. A 24, 645 (1967).

${ }^{10}$ R. E. Jones Jr., H. R. Shanks, D. K. Finnemore, and B. Morosin, Phys. Rev. B 6, 835 (1972).

${ }^{11}$ I. Guillamón, H. Suderow, S. Vieira, L. Cario, P. Diener, and P. Rodière, Phys. Rev. Lett. 101, 166407 (2008).

${ }^{12}$ I. Guillamón, H. Suderow, F. Guinea, and S. Vieira, Phys. Rev. B 77, 134505 (2008).

${ }^{13}$ R. Schuster, R. Kraus, M. Knupfer, H. Berger, and B. Büchner, Phys. Rev. B 79, 045134 (2009).

${ }^{14}$ J. van Wezel, R. Schuster, A. König, M. Knupfer, J. van den Brink, H. Berger, and B. Büchner, Phys. Rev. Lett. 107, 176404 (2011).

${ }^{15}$ R. Manzke, G. Crecelius, J. Fink, and R. Schöllhorn, Solid State Commun. 40, 103 (1981).

${ }^{16}$ S. L. Adler, Phys. Rev. 126, 413 (1962).

${ }^{17}$ A. vom Felde, J. Fink, Th. Büche, B. Scheerer, and N. Nücker, Europhys. Lett. 4, 1037 (1987).

${ }^{18}$ A. vom Felde, J. Spösser-Prou, and J. Fink, Phys. Rev. B 40, 10181 (1989).

${ }^{19}$ F. Aryasetiawan and K. Karlsson, Phys. Rev. Lett. 73, 1679 (1994).

${ }^{20}$ A. Fleszar, R. Stumpf, and A. G. Eguiluz, Phys. Rev. B 55, 2068 (1997).

${ }^{21}$ M. D. Johannes, I. I. Mazin, and C. A. Howells, Phys. Rev. B 73, 205102 (2006).

${ }^{22}$ E. E. Krasovskii, W. Schattke, V. N. Strocov, and R. Claessen, Phys. Rev. B 66, 235403 (2002).

${ }^{23}$ A. L. Fetter, Ann. Phys. 88, 1 (1974).

${ }^{24}$ S. Das Sarma and J. J. Quinn, Phys. Rev. B 25, 7603 (1982).

${ }^{25}$ A. C. Tselis and J. J. Quinn, Phys. Rev. B 29, 3318 (1984).

${ }^{26}$ J. K. Jain and P. B. Allen, Phys. Rev. Lett. 54, 2437 (1985).

${ }^{27}$ G. F. Giuliani and J. J. Quinn, Phys. Rev. Lett. 51, 919 (1983).
${ }^{28}$ P. Hawrylak, J.-W. Wu, and J. J. Quinn, Phys. Rev. B 32, 4272 (1985).

${ }^{29}$ V. Z. Kresin and H. Morawitz, Phys. Rev. B 37, 7854 (1988).

${ }^{30}$ V. Z. Kresin and H. Morawitz, Phys. Rev. B 43, 2691 (1991).

${ }^{31}$ V. M. Silkin, A. Balassis, P. M. Echenique, and E. V. Chulkov, Phys. Rev. B 80, 054521 (2009).

${ }^{32}$ A. Bill, H. Morawitz, and V. Z. Kresin, Phys. Rev. B 66, 100501 (2002).

${ }^{33}$ A. Bill, H. Morawitz, and V. Z. Kresin, Phys. Rev. B 68, 144519 (2003).

${ }^{34}$ J. P. Echeverry, E. V. Chulkov, P. M. Echenique, and V. M. Silkin, Phys. Rev. B 85, 205135 (2012).

${ }^{35}$ E. Runge and E. K. U. Gross, Phys. Rev. Lett. 52, 997 (1984).

${ }^{36}$ M. Petersilka, U. J. Gossmann, and E. K. U. Gross, Phys. Rev. Lett. 76, 1212 (1996)

${ }^{37}$ E. K. U. Gross and W. Kohn, Phys. Rev. Lett. 55, 2850 (1985).

${ }^{38}$ F. Aryasetiawan and O. Gunnarsson, Phys. Rev. B 49, 16214 (1994).

${ }^{39}$ D. M. Ceperley and B. J. Alder, Phys. Rev. Lett. 45, 566 (1980).

${ }^{40}$ J. P. Perdew and A. Zunger, Phys. Rev. B 23, 5048 (1981).

${ }^{41}$ N. Troullier and J. L. Martins, Phys. Rev. B 43, 1993 (1991).

${ }^{42}$ M. Marezio, P. D. Dernier, A. Menth, and G. W. Hull Jr., J. Solid State Chem. 4, 425 (1972).

${ }^{43}$ R. Corcoran, P. Meeson, Y. Onuki, P.-A. Probst, M. Springford, K. Takita, H. Harima, G. Y. Guo, and B. L. Gyorffy, J. Phys.: Condens. Matter 6, 4479 (1994).

${ }^{44}$ K. Rossnagel, O. Seifarth, L. Kipp, M. Skibowski, D. Voß, P. Krüger, A. Mazur, and J. Pollmann, Phys. Rev. B 64, 235119 (2001).

${ }^{45}$ M. Calandra, I. I. Mazin, and F. Mauri, Phys. Rev. B 80, 241108(R) (2009).

${ }^{46}$ Th. Straub, Th. Finteis, R. Claessen, P. Steiner, S. Hüfner, P. Blaha, C. S. Oglesby, and E. Bucher, Phys. Rev. Lett. 82, 4504 (1999).

${ }^{47}$ T. Valla, A. V. Fedorov, P. D. Johnson, P-A. Glans, C. McGuinness, K. E. Smith, E. Y. Andrei, and H. Berger, Phys. Rev. Lett. 92, 086401 (2004).

${ }^{48}$ D. S. Inosov, V. B. Zabolotnyy, D. V. Evtushinsky, A. A. Kordyuk, B. Büchner, R. Follath, H. Berger, and S. V. Borisenko, New J. Phys. 10, 125027 (2008).

${ }^{49}$ D. W. Shen, Y. Zhang, L. X. Yang, J. Wei, H. W. Ou, J. K. Dong, B. P. Xie, C. He, J. F. Zhao, B. Zhou, M. Arita, K. Shimada, H. Namatame, M. Taniguchi, J. Shi, and D. L. Feng, Phys. Rev. Lett. 101, 226406 (2008). 
${ }^{50}$ S. V. Borisenko, A. A. Kordyuk, V. B. Zabolotnyy, D. S. Inosov, D. Evtushinsky, B. Büchner, A. N. Yaresko, A. Varykhalov, R. Follath, W. Eberhardt, L. Patthey, and H. Berger, Phys. Rev. Lett. 102, 166402 (2009).

${ }^{51}$ M. G. Bell and W. Y. Liang, Adv. Phys. 25, 53 (1976).

${ }^{52}$ I. Errea, A. Rodriguez-Prieto, B. Rousseau, V. M. Silkin, and A. Bergara, Phys. Rev. B 81, 205105 (2010).

${ }^{53}$ V. P. Zhukov, V. M. Silkin, E. V. Chulkov, and P. M. Echenique, Phys. Rev. B 64, 180507(R) (2001).

${ }^{54}$ W. Ku, W. E. Pickett, R. T. Scalettar, and A. G. Eguiluz, Phys. Rev. Lett. 88, 057001 (2002).

${ }^{55}$ Y. Q. Cai, P. C. Chow, O. D. Restrepo, Y. Takano, K. Togano, H. Kito, H. Ishii, C. C. Chen, K. S. Liang, C. T. Chen, S. Tsuda, S. Shin, C. C. Kao, W. Ku, and A. G. Eguiluz, Phys. Rev. Lett. 97, 176402 (2006).

${ }^{56}$ A. Balassis, E. V. Chulkov, P. M. Echenique, and V. M. Silkin, Phys. Rev. B 78, 224502 (2008).
${ }^{57}$ With present calculation setup we were able to trace the dispersion of the low-energy plasmon for the momentum transfers along the (001) direction down to energy of $\sim 0.1 \mathrm{eV}$ at small momenta. Much more detailed calculations are required in order to confirm or disregard its existence at smaller energies.

${ }^{58}$ V. M. Silkin, I. P. Chernov, Yu. M. Koroteev, and E. V. Chulkov, Phys. Rev. B 80, 245114 (2009).

${ }^{59}$ D. Pines, Can. J. Phys. 34, 1379 (1956).

${ }^{60}$ H. Fröhlich, J. Phys. C 1, 544 (1968).

${ }^{61}$ E. A. Pashitskii and V. I. Pentegov, Low Temp. Phys. 34, 113 (2008).

${ }^{62}$ B. Geilikman, Usp. Fiz. Nauk 109, 665 (1973) [Sov. Phys. Usp. 16, 17 (1973)].

${ }^{63}$ J. Ihm, M. L. Cohen, and S. F. Tuan, Phys. Rev. B 23, 3258 (1981).

${ }^{64}$ J. Ruvalds, Adv. Phys. 30, 677 (1981).

${ }^{65} \mathrm{D}$. Pines and P. Nozières, The Theory of Quantum Liquids (Benjamin, New York, 1966). 